\title{
DYNAMIC PROPERTIES OF CELLULAR NEURAL NETWORKS ${ }^{1}$
}

\author{
ANGELA SLAVOVA ${ }^{2}$ \\ Center of Mathematics \\ Technical University \\ Russe 7017, BULGARIA
}

\begin{abstract}
Dynamic behavior of a new class of information-processing systems called Cellular Neural Networks is investigated. In this paper we introduce a small parameter in the state equation of a cellular neural network and we seek for periodic phenomena. New approach is used for proving stability of a cellular neural network by constructing Lyapunov's majorizing equations. This algorithm is helpful for finding a map from initial continuous state space of a cellular neural network into discrete output. A comparison between cellular neural networks and cellular automata is made.
\end{abstract}

Key words: Cellular neural networks, cellular automata, Lyapunov's majorant, finite majorizing equations. $58 \mathrm{~F} 10$.

AMS (MOS) subject classification: 92B20, 58F22, 34C35,

\section{INTRODUCTION}

Cellular Neural Networks present a new class of information-processing systems. It is a large-scale nonlinear analog circuit which process signals in real time. The basic circuit unit of a cellular neural network is called a cell, an analog processor element which contains linear and nonlinear circuit elements, typically - linear capacitors, linear resistors, linear and nonlinear controlled sources, and independent sources [1, fig.3] . The architecture of typical cellular neural networks is similar to that found in cellular automata $[7,8]$ : any cell in a cellular neural network is connected only to its neighbor cells. Adjacent cells can interact directly with each other. Cells not directly connected may affect each other indirectly, because of the propagation effects of the continuous-time dynamics of the networks. The main difference between cellular automata and cellular neural networks is in their dynamic behavior. A cellular neural network is a continuous time dynamical system. A cellular automaton is a discrete time dynamical system.

\footnotetext{
${ }^{1}$ Received: February, 1993, Revised: May, 1993.

${ }^{2}$ Current address: Florida Institute of Technology, Melbourne, Florida 32901, U.S.A., under Fulbright Scholarship.
} 
Some theoretical results concerning the dynamic range and the steady-state behavior of cellular neural networks have been presented in [1]. The dynamical system equations describing a typical cellular neural network cell [1, fig.3] consists of the following equations and constraints:

1) State Equation:

$$
\begin{aligned}
C \frac{d x_{i j}}{d t}= & -\frac{1}{R_{x}} v_{x i j}(t)+\sum_{C(k, l) \in N_{r}(i, j)} A(i, j ; k, l) v_{y k l}(t)+ \\
& +\sum_{C(k, l) \in N_{r}(i, j)} B(i, j ; k, l) v_{u k l}(t)+I \\
& 1 \leq i \leq M, 1 \leq j \leq N
\end{aligned}
$$

where the $i$ th row and $j$ th column cell is indicated as $C(i, j)$; by definition the $r$-neighborhood $N_{r}$ of radius $r$ of a cell, $C(i, j)$, in a cellular neural network is [1]

$$
N_{r}(i, j)=\{C(k, l) \mid \max \{|k-i|,|l-j|\} \leq r, 1 \leq k \leq M, 1 \leq l \leq N\}
$$

$v_{x i j}, v_{y i j}, v_{u i j}$ refer to the state, output and input voltage of a cell $C(i, j) ; C, R_{x}$ are fixed values of a linear capacitor and a linear resistor in the cell; $I$ is an independent voltage; $A(i, j ; k, l)$ is a feedback operator and $B(i, j ; k, l)$ - control operator, for which $I_{x y}(i, j ; k, l)=A(i, j ;, k, l) v_{y k l}$ and $I_{x u}(i, j ; k, l)=B(i, j ; k, l) v_{u k l}\left(I_{x y}(i, j ; k, l)\right.$ and $I_{x u}(i, j ; k, l)$ are linear voltage-controlled sources for all $C(i, j) \in N(i, j))$.

2) Output Equation:

$$
\begin{gathered}
f\left(v_{x i j}\right)=v_{y i j}, \\
v_{y i j}(t)=\left\{\begin{array}{ccc}
0, & v_{x i j}(t)<0, & 1 \leq i \leq M \\
v_{x i j}(t), & 0 \leq v_{x i j} \leq 1, & 1 \leq j \leq N \\
1, & v_{x i j}(t)>1 &
\end{array}\right.
\end{gathered}
$$

where function $f$ is piecewise-linear.

Function $f$ can be approximated within any precision by a smooth $\left(C^{1}\right)$ sigmoid function, which help us in analytical proofs, since $C^{1}$ is more convenient to work with.

3) Input Equation:

$$
v_{u i j}=E_{i j}, \quad 1 \leq i \leq M, 1 \leq j \leq M .
$$

4) Constraint Equations:

$$
0 \leq v_{x i j}(0) \leq 1
$$




$$
\left|v_{u i j}\right| \leq 1
$$

5) Parameter Assumptions:

$$
\begin{gathered}
A(i, j ; k, l)=A(k, l ; i, j) \\
C>0, R_{x}>0 .
\end{gathered}
$$

As mentioned above in [1] it is proved that cellular neural networks, described by the equations (1-7) must always converge to a constant steady-state after the transient has decayed to zero. Moreover, it is obtained that cellular neural networks have binary-valued outputs, which have applications in image-processing [2]. These properties of cellular neural networks imply that the circuit will not oscillate and become chaotic.

Our interests are in obtaining some periodic or chaotic behavior of a cellular neural network. For this purpose we introduce a small parameter $\mu$ in front of function $f$. Then the state equation (1) can be rewritten in the following form:

$$
C \frac{d v}{d t}=-\frac{1}{R_{x}} v+\mu A f(v)+I
$$

where $v=v_{x i j}(t), A=A(i, j ; k, l), f(v)=f\left(v_{x i j}\right)$, and we also assume that $B(i, j ; k, l)=0$. It is convenient for our further analysis and comparison of cellular neural networks to cellular automata.

One of the most popular techniques for analyzing the stability of dynamical nonlinear circuits is Lyapunov's method by defining an appropriate Lyapunov function for cellular neural networks [1].

Since we introduce a small parameter $\mu$ in equation (8), our approach will differ from the above. We will use the Lyapunov's majorizing equations method to investigate the convergence properties of cellular neural networks, described by state equation (8). Moreover, we will seek for the periodic solutions of (8) and dynamic range of cellular neural network. Stability of equilibrium points of cellular neural networks will change as an effect of a small parameter $\mu$ and we can estimate the upper bound of the interval of values of $\mu$, in which periodic solution exists. 


\section{PRELIMINARIES}

Lyapunov's majorizing equations method can be applied for the operator system of quite general kind with bounded linear operators:

$$
x=L F(x, t, \mu)
$$

where the function $F$ belongs to the class $C^{1}$ with respect to $x(t, \mu)$ and to the class $C$ with respect to $t, \mu$. $L$ is a linear and bounded operator. Suppose also that :

$$
F(0, t, 0)=0, \frac{\partial F(0, t, 0)}{\partial x}=0 .
$$

From the boundedness of the operator $L$ it follows the existence of a finite constant $\rho>0$, such that the following inequality is satisfied for any function $\varphi \in C$ :

$$
\|L \varphi(t)\| \leq \rho\|\varphi(t)\|, t \in[0, T] .
$$

We introduce the class $\Omega$ of functions $\Phi(\alpha, \mu)$ such that:

i) $\Phi(\alpha, \mu)$ is a function of $\alpha>0$, belonging to the class $C^{1}[\alpha]$ for $|\alpha| \leq R(R$ is a constant), and class $C[\mu]$;

ii) $\Phi(\alpha, \mu)$ is positive for $\alpha>0, \mu>0$, monotone increasing with respect to all arguments and convex in $\alpha$ in the case of nonlinearity in $\alpha$.

Definition 1. A Lyapunov's majorant with respect to the function $F(x, t, \mu)$ is a function $\Phi(\alpha, \mu)$ of class $\Omega$ in the domain $|\alpha| \leq R, 0 \leq \mu \leq \mu_{*}$, such that for $|\alpha| \leq R$ and $|x| \leq \alpha$ the following inequalities are valid:

$$
\begin{gathered}
\|F(x, t, \mu)\| \leq \Phi(\alpha, \mu), \\
\left\|\frac{\partial F(x, t, \mu)}{\partial x}\right\| \leq \frac{\partial \Phi(\alpha, \mu)}{\partial \alpha} .
\end{gathered}
$$

Lyapunov's majorizing equation is as follows:

$$
\alpha=\rho \Phi(\alpha, \mu)
$$

Due to the properties of Lyapunov's majorizing equations [3], we have the following basic theorem:

Theorem 1: If system (12) has for $\mu \in\left[0, \mu_{*}\right]$ a solution $\alpha(\mu) \in C\left[0, \mu_{*}\right]$, which is positive for $\mu>0$ and such that $\alpha(0)=0$ and $\left\|\alpha\left(\mu_{*}\right)\right\| \leq R$, then the successive approximations:

$$
x_{k}=L F\left(x_{k-1}, t, \mu\right), k=1,2, \ldots
$$




$$
x_{0} \equiv 0
$$

converge for $t \in[0, T], \mu \in\left[0, \mu_{*}\right]$ to the solution $x(t, \mu)$ of $(9)$, which is unique.

\section{DYNAMIC BEHAVIOR OF CELLULAR NEURAL NETWORKS}

If we consider function $f(v)$ from the equation (2), we have four possible cases for the state of a cell $C(i, j)$ :

1) $\quad v_{x i j}(t)<0$,

2) $\quad 0 \leq v_{x i j}(t) \leq 1$ and $\frac{d v_{x i j}}{d t}=0$,

3) $0 \leq v_{x i j}(t) \leq 1$ and $\frac{d v_{x i j}}{d t} \neq 0$,

4) $\quad v_{x i j}(t)>1$.

When $v_{x i j}(t)<0$, then $v_{y i j}(t)=0$ for all $t>0$, no matter if $v_{x i j}(t)=$ const. or $v_{x i j}(t) \neq$ const., which corresponds to the first case. When $v_{x i j}>1$, then $v_{y i j}(t)=1$ for all $t>0$, so we have the fourth case. If $0 \leq v_{x i j} \leq 1$, then $v_{y i j}(t)=v_{x i}(t)$. In the third case $v_{x i j}(t) \neq$ const. and we can expect some periodic behavior or oscillation.

Now let us consider equation (8), which can be rewritten in the following form:

$$
\frac{d v}{d t}=M v+g(v, t, \mu)
$$

where $M=-\frac{1}{C R_{x}}, g(v, t, \mu)=\mu \frac{A}{C} f(v)+\frac{1}{C} I$.

Assume that $g(v, t, \mu)$ is a periodic function in $t$ with period $T$, in other words we consider the third case. Function $g(v, t, \mu)$ satisfies also the following conditions:

$$
g(0, t, 0)=0, \frac{\partial g(0, t, 0)}{\partial v}=0 .
$$

Let us consider an auxiliary linear nonhomogeneous equation for equation (13):

$$
\frac{d v}{d t}=M v+\varphi(t)
$$

where $\varphi(t)$ is an arbitrary, periodic function with period $T$. Its solution is

$$
v(t)=\left[e^{-M T}-E\right]^{-1} \int_{t}^{t+T} e^{M(t-s)} \varphi(s) d s .
$$

This formula defines $v$ as the result of the application of an operator $L$ to the function $\varphi(t)$, i.e.

$$
v(t)=L \varphi(t), t \in[0, T]
$$


The operator $L$ is linear and bounded. Therefore there exists a constant $\rho$, such that inequality (11) will be satisfied. If we go back to equation (12), the following can be obtained:

$$
v(t, \mu)=L g(v, t, \mu)
$$

which is an operator equation of type (9), equivalent to equation (13).

Now we can write Lyapunov's majorizing equation (18) in the certain domain $|v| \leq 1$ :

$$
\alpha=\rho \Phi(\alpha, \mu)
$$

where $\Phi(\alpha, \mu)$ is a Lyapunov's majorant for the function $g(v, t, \mu)$ in the form:

$$
\Phi(\alpha, \mu)=\mu \frac{A}{C} \alpha+\frac{1}{C} I, \alpha \geq|v| .
$$

According to the properties of Lyapunov's majorizing equations [3] we can conclude that for $\mu \in\left[0, \mu_{*}\right)$ the solution $\alpha(\mu)$ of $(20)$ is positive and $\alpha(0)=0$. Therefore by using Theorem 1 , the following theorem has been proved:

Theorem 2: $\quad$ Suppose that equation (19) in the domain $|v| \leq 1$ has a positive solution $\alpha(\mu)$ for $0 \leq \mu<\mu_{*}$ and $\alpha\left(\mu_{*}\right) \leq 1$. Then equation (13) has for $0 \leq \mu<\mu_{*}$ a periodic solution $v=v(t, \mu)$, with period $T$, which is unique in the class $C[\mu]$. This solution can be found by the simple iterations:

$$
\begin{gathered}
v_{k}=L_{g}\left(v_{k-1}, t, \mu\right), k=1,2, \ldots \\
v_{0}=v(0)
\end{gathered}
$$

\section{Remarks and Conclusions:}

1. From the properties of Lyapunov's majorizing equations [3] and since function $\Phi(\alpha, \mu)$ is linear, it follows that solution $\alpha=\alpha(\mu)$ will exist on the half-open interval $0 \leq \mu<\mu_{*^{*}}$. The solution $\alpha=\alpha(\mu)$ may be unbounded for $\mu \rightarrow \mu_{*^{*}}$. If we consider the plot of curves $y=\Phi(\alpha, \mu)$ for $\mu\left\langle\mu_{*}, \mu=\mu_{*}, \mu>\mu_{*}\right.$ in the plane $(\alpha, y)$ and the plot of the straight line $y=\alpha$, we can see that curve $\Phi(\alpha, \mu)$ for $\mu>\mu_{*}$ diverges.

2. Small parameter $\mu$ affects dynamic range of a cell, in other words if $\mu>\mu_{*}$, state voltage $v_{x i j}(t)$ will be unbounded and this implies in different unpredictable boundary effects between neighbor cells. If $\mu \in\left[0, \mu_{*}\right)$, state voltage $v_{x i j}(t)$ is bounded (from Theorem 2) and periodic, therefore output $v_{y i j}(t)=v_{x i j}, 0 \leq v_{x i j} \leq 1$ will have periodic behavior. 
3. Consider iterations (21) and fix $t=\tau, \mu=\mu_{0}, \mu_{0} \in\left[0, \mu_{*}\right)$. Then

$$
\begin{gathered}
v_{k}=L_{\tau} g\left(v_{k-1}, \mu_{0}\right), \quad k=1,2, \ldots \\
v_{0}=v(0),
\end{gathered}
$$

gives the dynamic rules of a cellular neural network. Therefore, we can use cellular neural networks to obtain a dynamic transform of an initial state at any time $t$. In the special cases when $t \rightarrow \infty$ and state variable $v_{x i j}$ is a constant, the output $v_{y i j}$ tends to either 0 or 1 , (see equation (2)), which are limit points for a cellular neural network.

4. In [1] it is shown that if $A(i, j ; k, l)>\frac{1}{R_{x}}$ then each cell of a cellular neural network must settle at a stable equilibrium point after the transient has decayed to zero. But in our case we have $\mu A(i, j ; k, l) \ngtr \frac{1}{R_{x}}$, which implies oscillation phenomena [4].

\section{STABILITY OF CELLULAR NEURAL NETWORKS}

The physical idea of stability is closely related to a "bounded" response of the dynamical system, when it is driven by an external input. A mathematical definition of stability is based on the "boundedness" of the solutions of differential equation [5,6]. This gives rise to many definitions of stability. For our cellular neural networks, in view of the nearest neighborhood interactive property, we will define the stability for all system equilibrium points by first determining the stable cell equilibrium states and then using the neighbor interactive rules to find the corresponding system equilibrium points.

From the dynamical point of view, if the system is disturbed and is displaced slightly from the equilibrium state, several situations are possible. If the trajectory of a cellular neural network starting from initial state remains near equilibrium state, then cellular neural network is said to be stable. If it tends to return to the equilibrium state, cellular neural network is said to be asymptotically stable.

Let us consider equation (8) :

$$
C \frac{d v_{x i j}}{d t}=-\frac{1}{R_{x}} v_{x i j}+\mu A(i, j ; k, l) f\left(v_{x i j}\right)+I,
$$

or in the form

$$
\frac{d v_{x i j}}{d t}=g_{1}\left(v_{x i j}, t, \mu\right)
$$

By definition the equilibrium points for system (23), will be $v_{x i j}^{*}$, for which $g_{1}\left(v_{x i j}^{*}, t, \mu\right)=0$. 
Therefore in $0 \leq v_{x i j}(t) \leq 1$, the equilibrium points of a typical cell of a cellular neural network $C(i, j)$ are defined as :

$$
v_{x i j}^{*}(t)=\frac{I R_{x}}{1-\mu A R_{x}}, 1 \leq i \leq M, 1 \leq j \leq N
$$

Then we can define stable system equilibrium points of a cellular neural network, which describe its global dynamic behavior.

Definition 2: $\quad$ Stable system equilibrium point of a cellular neural network is

a state vector with components $v_{x i j}^{*}(t), 1 \leq i \leq M, 1 \leq j \leq N$, for which $\mu<\frac{1}{A(i, j ; k, l) R_{x}}$ and $\lim _{t \rightarrow \infty} v_{y i j}(t)=0$ or 1 .

From the above definition we can see that if we change the parameter, it will change the equilibrium points. Since any stable system equilibrium point is a limit point of a set of trajectories of the corresponding differential equation (8), such an attracting limit point is said to have a basin of attraction [5]. Therefore parameter $\mu$ can affect the stability of a cellular neural network, in sense that different limit points and basins of attraction will be obtained for different values of small parameter $\mu$. So, if we consider the initial state space as $[0,1]^{M \times N}$ and the output space as $\{0,1\}^{M \times N}$, then cellular neural network can be used to map an initial state of a system into one of many distinct stable equilibrium points and this map, defined by (22), will depend on $\mu$. In other words we have:

$$
F_{\mu}:[0,1]^{M \times N} \rightarrow\{0,1\}^{M \times N}
$$

which gives us the dynamic behavior of cellular neural networks.

\section{SIMILARITY BETWEEN CELLULAR NEURAL NETWORKS AND CELLULAR AUTOMATA}

In Section 3 we reduced state equation (8) of a cellular neural network to the operator equation (18). According to Theorem 2, state voltage $v_{x i j}(t)$ of cell $C(i, j)$ can be found as a boundary of the iterations:

$$
\begin{gathered}
v_{k}=L g\left(v_{k-1}, t, \mu\right), k=1,2, \ldots \\
v_{0}=v(0)
\end{gathered}
$$

where $v=v_{x i j}(t), 1 \leq i \leq M, 1 \leq j \leq N$. As stated in Remark 3 , if we fix $t=\tau$ and $\mu=\mu_{0} \in\left[0, \mu_{*}\right)$, we can obtain a dynamic transform of an initial state at any time $t$. 
Therefore the equation

$$
\begin{gathered}
v_{k}=L_{\tau} g\left(v_{k-1}, t, \mu\right), k=1,2, \ldots \\
v_{0}=v(0)
\end{gathered}
$$

gives the dynamic rules of the state variables of a cellular neural network. In other words, equation (26) gives the rules of interaction between cells in a cellular neural network. The output equation (2) of a cellular neural network can be rewritten in the form:

$$
v_{y i j}^{k}=\left\{\begin{array}{cc}
0, & v_{x i j}^{k}<0 \\
v_{x i j}^{k}, & 0 \leq v_{x i j}^{k} \leq 1 \\
1, & v_{x i j}^{k}>1
\end{array}\right.
$$

or

$$
v_{y i j}^{k}=\Psi\left\{v_{x i j}^{k-1}, v_{y k l}^{k-1} \text { for all } C(k, l) \in N_{r}(i, j)\right\}
$$

As we said before the structure of the cellular neural networks is similar to that of cellular automata. A typical equation of a two-dimensional cellular automaton is ([7])

$$
a_{i j}(n+1)=\Phi\left[a_{k l}(n) \text { for all } C(k, l) \in N_{r}(i, j)\right] .
$$

Comparing equation (27) and equation (28) we can see a similarity between them. Therefore we can use cellular automata theory to study dynamic behavior of the cellular neural networks. It is well known [7], that cellular automata may be considered as discrete dynamical system. In almost all cases, cellular automata evolution is irreversible. Trajectories in the configuration space for cellular automata therefore merge with time, and after many time steps, trajectories starting from almost all initial states become concentrated onto attractors. These attractors typically contain only a very small fraction of possible states. There are four classes of cellular automata, which characterize the attractors in cellular automaton evolution. The attractors in classes 1,2 and 3 are roughly analogous respectively to the limit points, limit cycles and chaotic attractors found in continuous dynamical systems [8].

\section{REFERENCES}

[1] Chua, L.O., Yang, L., Cellular neural networks: Theory, IEEE Trans. Circuits Syst. 35, (1988), 1257-1272.

[2] Chua, L.O., Yang, L., CNN: Applications, IEEE Trans. Circuits Syst. 35, (1988), 1273-1299. 
[3] Lyapunov, A.M., General Problem about the Stability of Motion, Gostekhizdat, Moscow, Russia 1950.

[4] Chua, L.O., Roska, T., Stability of a class of nonreciprocal CNN, IEEE Trans. Circuits Syst. 37, (1990), 1520-1527.

[5] Guckenheimer, J., Holmes, Ph., Nonlinear Oscillations, Dynamical Systems, and Bifurcations of Vector Fields, Springer 1983.

[6] LaSalle, J.P., The Stability of Dynamical Systems, SIAM, Philadelphia 1976.

[7] Packard, N.H., Wolfram, S., Two-dimensional cellular automata, J. of Stat. Phys. 38 (1985), 901-946.

[8] Wolfram, S., University and complexity in CA, Physica 10D, (1984), 1-35. 


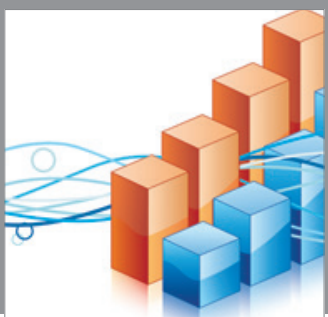

Advances in

Operations Research

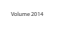

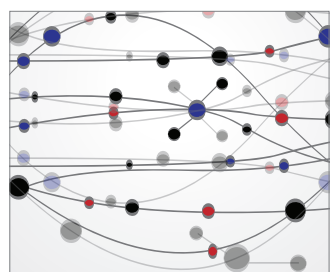

\section{The Scientific} World Journal
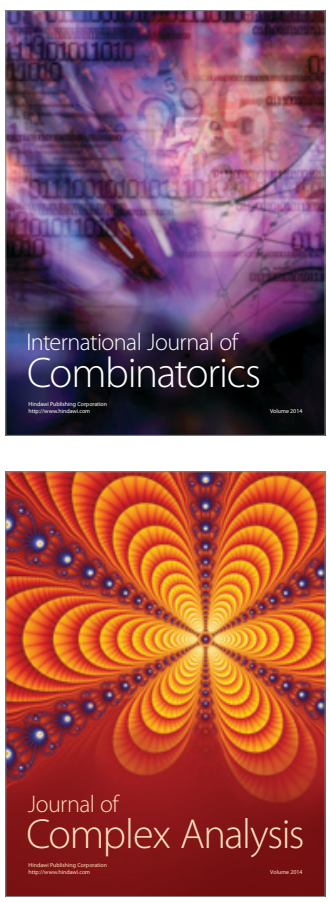

International Journal of

Mathematics and

Mathematical

Sciences
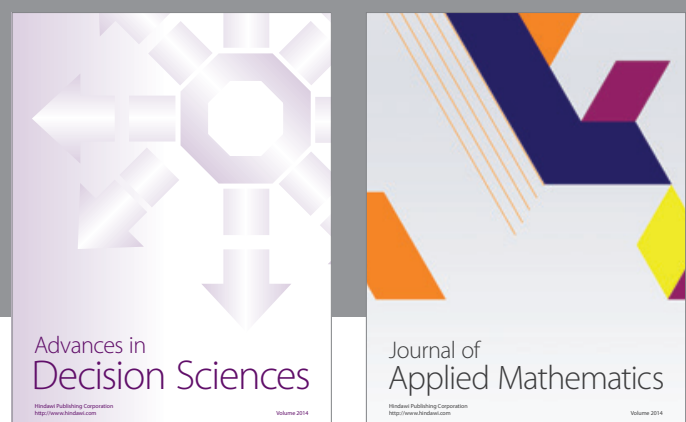

Journal of

Applied Mathematics
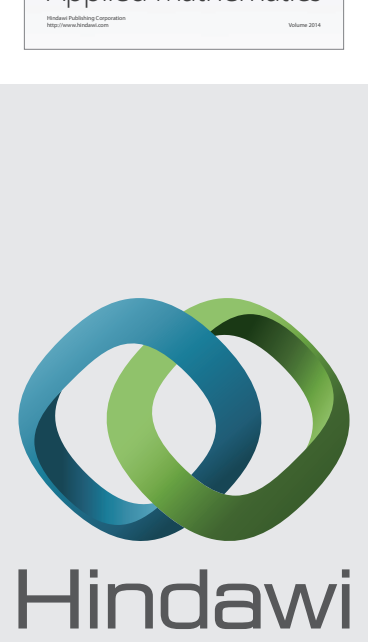

Submit your manuscripts at http://www.hindawi.com
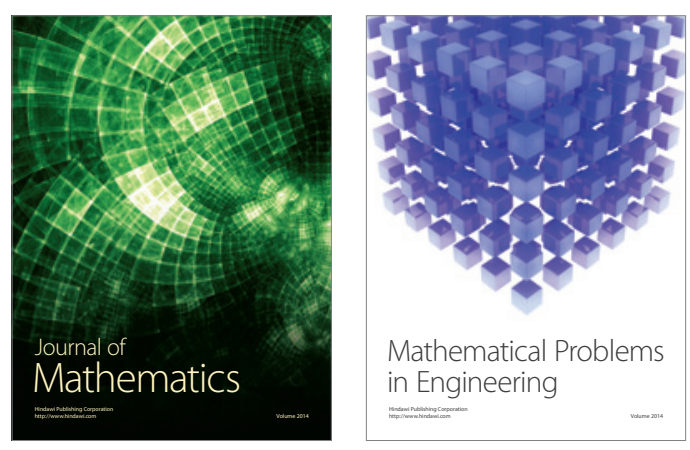

Mathematical Problems in Engineering
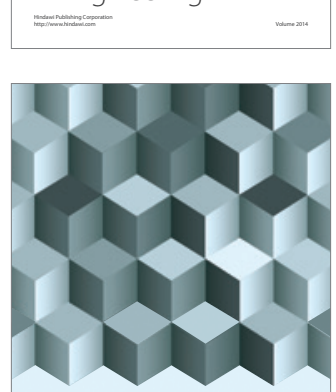

Journal of

Function Spaces
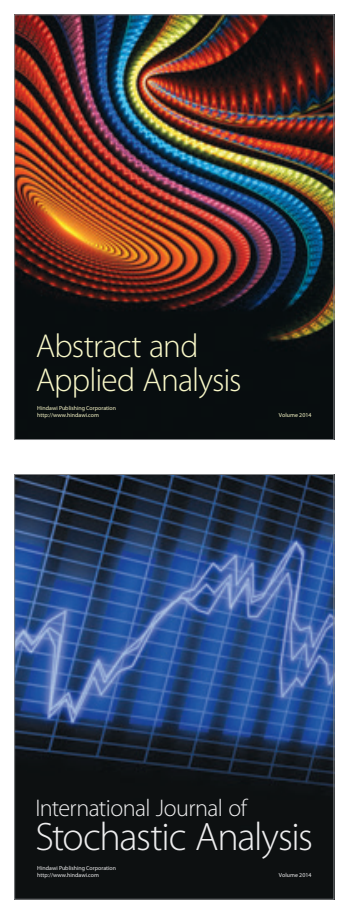

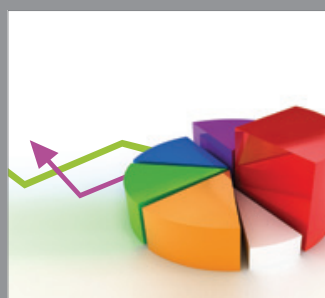

ournal of

Probability and Statistics

Promensencen
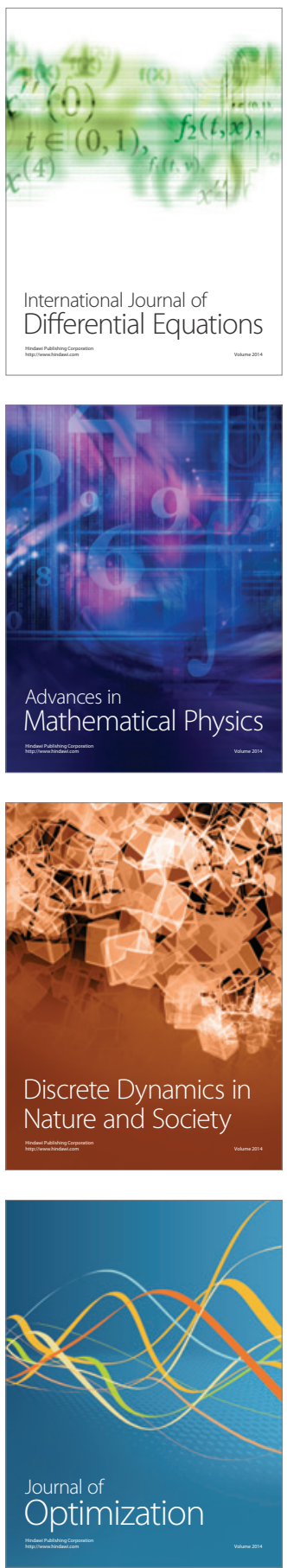